\title{
Equine Herpes Virus 1 and 4 Infections in equines: A Review
}

\author{
Jignesh Vala ${ }^{\text {* }}$, Manish Patel ${ }^{2}$, Saurabh Parmar ${ }^{1}$, Suresh Mavadiya ${ }^{3}$ and Sudhir Mehta ${ }^{3}$ \\ ${ }^{1}$ Veterinary Clinical Complex, ${ }^{3}$ Department of Veterinary Medicine, \\ Veterinary College, Navsari Agricultural University, Navsari, India \\ ${ }^{2}$ Department of Animal Science, College of Agriculture, \\ Navsari Agriculture Univesity, Waghai, India \\ *Corresponding author
}

\section{Keywords}

Equines, Herpes virus,

EHV 1, EHV 4, Rhinopneumonitis

\section{Article Info}

Accepted:

05 June 2020

Available Online:

10 July 2020

\section{A B S T R A C T}

EHV - 1 and 4 both viruses are most important causes of febrile rhinopneumonitis among equine and in some countries EHV - 1 is major factor responsible for abortion and myeloencephalopathy outbreaks. Inhalation of aerosolized viral particles provide major role in disease transmission and virus primarily have an effect on respiratory and uterine mucosa as well as spinal cord causing rhinopneumonitis, abortion and myeloencephalopathy. Previously virus isolation considered as a gold standard for diagnosis but with evolution of advanced molecular approach recently real time PCR is considered as a gold standard. Management of myeloencephalopathic cases is quiet difficult and required long term patience and strong will power. Prevention of these infectious diseases relies heavily on the use of vaccination.

\section{Introduction}

Herpes viruses are ubiquitous in nature having wide host range including insects, reptiles, amphibians as well as every species of mammals and birds. Around 14 herpes viruses in nature are present infecting every species of equidae family. Among these 14 herpes viruses infecting equines equine herpes virus (EHV) 1 and 4 are the most studied around the globe. EHV - 1 infection in equines is generally responsible for upper respiratory tract infection in young horses and abortion, usually in late gestation as well as myeloencephalopathy in adult horses. EHV 4 previously had been thought to be a subtype of EHV - 1 until 1981, but through molecular characterization techniques, the virus was recognized as distinct viral species causing upper respiratory tract infection commonly known as equine rhinopneumonitis. EHV - 1 and 4 are ubiquitous in equine population and 
both viruses have double stranded DNA genome consisting 145 to 150 kilo base encoding for 76 unique genes (Telford et al., 1998).

\section{Transmission}

Infection with EHV - 1 and 4 occurs through the respiratory tract by inhalation of the aerosolized infectious virus, nose-to-nose contact, or contact with fomites (Bell et al., 2006; Hussey and Landolt, 2015). Presence of EHV - 1 in stallion semen has also been reported (Fritsche and Borchers, 2011). The epidemiological features of EHV infections are: (i) high incidence of respiratory infection early in life with low morbidity, (ii) establishment of lifelong latency in a large proportion of infected equids, and (iii) frequent reactivation of latent virus with subsequent shedding, resulting in transmission to naive hosts (Allen et al., 2004; Hussey and Landolt, 2015). In general, latency and reactivation are the key factors of epidemiology of EHV - 1 and EHV - 4 infections and are responsible for ubiquitous distribution of these viruses in equine population.

\section{Pathogenesis}

Primarily EHV - 1 and 4 infection initiated by infecting respiratory epithelium causing distinct lesions on upper respiratory tract and viral shedding (Lunn et al., 2009). Cell to cell spread of EHV - 1 results in diffuse infection in respiratory lymph nodes, where further infection and replication in $\mathrm{T}$ lymphocytes and monocytes begin cell associated viremia, which can lasts till 9 to 23 days post infection (Wilsterman et al., 2011; Gryspeerdt et al., 2010). The cell associated viremia allows the virus to reach secondary sites of virus replication particularly the endothelial cells of pregnant uterus and central nervous system. (Hussey et al., 2013). Translocation of virus from the circulation in to placenta causing infection of endothelial cells of the endometrium leading to thrombosis, asculitis, microcotyledonary infarction and perivascular cuffing (Smith and Borchers, 2001).

Severe infection of endometrium leads to premature placental separation and abortion of fetus. The virus can also transfer across utero-placental barrier and affect fetal viscera leading to abortion with characteristic lesions of necrosis (Cullinane et al., 2006). Damage to the endothelial cells of microvasculature of central nervous system leads to initiation of inflammatory cascade, vasculitis, microthrombosis, extravasation of mononuclear cells resulting in perivascular cuffing and local hemorrhage (Pusterla and Hussey, 2014).

In contrast to EHV - 1, EHV - 4 infection is mainly limited to upper respiratory tract infection causing rhinopharyngitis and tracheobronchitis. Leukocyte associated viremia is rare in EHV - 4 infection as compare to EHV - 1 (Vandekerckhove et al., 2011).

\section{Clinical symptoms}

\section{Respiratory disease}

The respiratory disease caused by both viruses is characterized by short incubation period followed by temporary elevation of body temperature, depression, inappatence, watery to purulent nasal discharge depending on secondary bacterial infection, enlarged submendibular lymph nodes and rarely ocular discharge (Patel and Heldens, 2005). Typical respiratory form predominantly seen in young horses between weaning and $2-3$ years of age (Gilkerson et al., 2015).

As a result of earlier infection with EHV - 1 and 4 , older horses generally show reduced 
duration and less severe respiratory tract disease (Wood et al., 2007).

\section{Abortion}

EHV - 1 infected pregnant mares usually abort late in gestation (8 to 11 months) without showing any prior clinical signs. The placenta is expelled with fetus, which has died from asphyxia or died shortly after birth (Pustrela and Hussey, 2014). Sporadic abortion in individual mares is more common but abortion outbreak in group of mares depend on management, degree of immunity, virulence of virus, various stress factors and number of exposed susceptible mares in advanced pregnancy (Swerczek and Dennis, 2006)

\section{Myeloencephalopathy}

The sudden onset of ataxia, paresis and urinary incontinence, the involvement of multiple horses on the premises, and a recent history of fever, abortion or respiratory disease in affected horse populations are typical features of EHM. Inception of myeloencephalopathy typically occurs suddenly between 6 to 10 days and severity reaches a peak within next 2 to 3 days (Pusterla and Hussey, 2014). Commonly, the caudal part of spinal cord is more severely affected, causing hind quarter weakness, bladder dysfunction and low sensitivity in perineal area.

\section{Disease in neonatal foals}

EHV - 1 infected foals may born weak, depressed and die within 3 days with severe respiratory distress. Some foals may born healthy and may survive for few weeks followed by secondary bacterial and/or viral infection. Such foals become pyrexic, leukopenic, hypoxic due to severe respiratory distress (Swerczek and Dennis, 2006)

\section{Diagnosis}

Despite a thorough anamnesis and a detailed clinical examination, it is usually not possible to diagnose any of the diseases associated with EHVs with certainty. Up to previous century, virus isolation was remains the "gold standard" for laboratory diagnosis of EHV infections and provides unequivocal evidence of the presence of infectious virus in clinical samples such as respiratory tract, blood, fetal and placental tissue samples (Lunn et al., 2009; Slater, 2014; Hussey and Landolt, 2015).

But with evolution of more recent molecular techniques, PCR-based assays have become key tools in the diagnosis of EHV infections. Positive PCR results can be obtained when virus isolation is negative due to inactivation of the virus or latent stage of the virus. With the use of specific primers, PCR can yield fast and accurate qualitative results. Several conventional PCR tests have been developed for the detection of DNA of the EHVs, with different type-specific primers capable of distinguishing between the different EHVs (Hue et al., 2014). The techniques especially real-time PCR has been extensively described in the literature (Hussey et al., 2013; Diallo et al., 2007; Quinilivan et al., 2005).

Immunofluorescence (IF) tests can be used to demonstrate viral antigens in frozen tissue samples rapidly and with high sensitivity and specificity (Slater, 2014). Cerebrospinal fluid analysis from equids with EHM often reveals xanthochromia and increased protein concentrations, reflecting vasculitis and protein leakage into the cerebrospinal fluid (Van Maanen, 2002). These cerebrospinal fluid changes, in conjunction with characteristic clinical signs, are suggestive, but not diagnostic, of EHM (Slater, 2014).

Serological assessment is the ideal tool to gain a retrospective diagnosis of EHV 
infections and forms a valuable part of longitudinal surveillance. Serologic testing which demonstrates a 4-fold or greater increase in serum antibody titer, by serum neutralizing $(\mathrm{SN})$ or complement-fixation (CF) tests, on acute and convalescent samples collected 7-21 days apart provides presumptive evidence of an EHV-1 infection (Lunn et al., 2009). CF antibodies are shortlived, usually becoming undetectable by 3 months after infection (Kydd et al., 2006). The CF antibody test is therefore useful as an indicator of recent EHV-1 infection and during outbreaks of disease. In contrast, because of the longevity of $\mathrm{SN}$ antibodies (persisting for more than 9 months), $\mathrm{SN}$ test is important for disease prevalence surveys (Kydd et al., 2006). However, neither SN nor $\mathrm{CF}$ test can distinguish antibodies to EHV-1 and EHV-4 (Slater, 2014). A commercially available enzyme-linked immunosorbent assay (ELISA), measuring antibodies directed against EHV $\mathrm{gG}$, is capable of differentiating between EHV-1 and EHV-4 infection (Lunn et al., 2009; Slater, 2014).

\section{Treatment and prognosis}

EHV - 4 infection is self limiting and mostly affects the upper the upper respiratory tract. On other hand, management of EHV - 1 infection is quiet lengthy and difficult especially in outbreak of abortion and myeloencephalopathy. The management should be directed to provide a good hygiene and judicious use of sling in cases of myeloencephalopathy. An indwelling Foley catheter can be attached to other fluid removing tube to prevent urinary scalding in bladder dysfunction. The antiviral drug acyclovir can be used at the dose rate of 10 $\mathrm{mg} / \mathrm{kg}$ orally for five days in cases of myeloencephalopathy. As a symptomatic treatment use of NSAIDs like phenylbutazone or flunixin meglumine or steroid like dexamethazone or prednisolone are useful
(Reed and Torbio, 2004). They also suggested use of intravenous dimethyl sulphoxide at the dose rate of $0.9 \mathrm{gm} / \mathrm{kg}$ as a $10 \%$ solution. The prognosis is mostly guarded mostly depends on immune status of animal. But, it may turn to favourable if owner have will power and patience with long term management.

\section{Control and prevention}

EHV - 1 disease control program has three common goals (1) prevention of viral entries in to premises; (2) limiting the extent of spread and severity of clinical disease once EHV - 1enters into premises; and (3) limiting the viral spread to adjacent premises (Slater, 2014). The source of EHV - 1 infection is usually horses that are unapparent carriers and the virus is transmitted by aerosol exposure, infected respiratory secretions, aborted foetus, placenta etc. Eliminating EHV infections from the equine population is very challenging because of the early establishment of life-long latency, despite the presence of maternal antibody and the ability of the virus to modulate or suppress the host immune system (Kydd et al., 2006). Prevention of infectious diseases relies heavily on the use of vaccination. The purpose of EHV-1 vaccination is (1) to minimize virus replication in the respiratory tract upon infection, thus limiting nasal shedding and the occurrence of respiratory disorders, and (2) to prevent the occurrence of abortion and/or nervous system disorders. Currently, modified live virus and inactivated vaccines are available for protection against EHV - 1 and 4 induced disease. These vaccines induce high titers of $\mathrm{CF}$ and $\mathrm{VN}$ antibody and appear to offer some protection against respiratory disease. They reduce the duration and titer of nasal virus shedding of virus (Goehring et al., 2010; Bresgen et al., 2012). However, they do not reliably block infection, the development of viremia or the establishment of latency. Indeed, EHM has 
been observed in horses regularly vaccinated against EHV-1 at 3 to 5 monthly intervals (Lunn et al., 2009; Hussey and Landolt, 2015). Currently, extensive research is continuing to develop improved vaccines such as recombinant vaccines and DNA vaccines against EHV-1 and EHV-4.

\section{References}

Allen G.P., Kydd J.H., Slater J.D. Smith K.C. (2004). Equid herpesvirus 1 and equid herpesvirus 4 infections., in: JAW Coetzer and RC Tustin (Ed.), Infectious Diseases of Livestock. Oxford Press, Cape Town, South Africa, pp. 829-859.

Bell S.A., Balasuriya U.B.R., Gardner I.A., Barry P.A., Wilson W.D., Ferraro G.L., MacLachlan N.J. (2006). Temporal detection of equine herpes virus infections of a cohort of mares and their foals. Vet Microbiol. 116:249-257.

Bresgen C., Lämmer M., Wagner B., Osterrieder N., Damiani A.M. (2012). Serological responses and clinical outcome after vaccination of mares and foals with equine herpesvirus type 1 and 4 (EHV-1 and EHV-4) vaccines. Vet. Microbiol. 160:9-16.

Cullinane A.A. Barr B., Bernard W., Duncan J.L., Mulcahy G., Smith I.M. Timoney J.F. (2006). Infectious diseases, in: The Equine Manual. Elsevier Ltd, pp. 1-111. Diallo I.S., Hewitson G., Wright L.L., Kelly M.A., Rodwell B.J. Corney B.G. (2007) Multiplex real-time PCR for the detection and differentiation of equid herpesvirus 1 (EHV-1) and equid herpesvirus 4 (EHV-4). Vet Microbiol 123:93-103.

Fritsche A.K. Borchers K. (2011). Detection of neuropathogenic strains of Equid Herpesvirus 1 (EHV-1) associated with abortions in Germany. Vet Microbiol. 147:176-180.

Gilkerson J.R., Bailey K.E., Diaz-Méndez A.
Hartley C.A. (2015). Update on Viral Diseases of the Equine Respiratory Tract. Vet Clin North Am Equine Prac. 31:91-104.

Goehring L.S., Landolt G.A., Morley P.S. (2010). Detection and Management of an Outbreak of Equine Herpesvirus Type 1 Infection and Associated Neurological Disease in a Veterinary Teaching Hospital. J Vet Int Med 24: 1176-1183.

Gryspeerdt, A., Vandekerckhove, A., Doorsselaere, J. Van and Nauwynck, H.J. (2011). Description of an unusually large outbreak of nervous system disorders caused by equine herpesvirus 1 (EHV1) in 2009 in Belgium. Vlaams Diergeneeskd Tijdschr. 80:147-153.

Hue E.S., Fortier G.D., Fortier C.I., Leon A.M., Richard E.A., Legrand L.J. Pronost S.L. (2014). Detection and quantitation of equid gamma herpesviruses (EHV-2, EHV-5) in nasal swabs using an accredited standardised quantitative PCR method. $J$ Virol Methods 198:18-25.

Hussey G.S. Landolt G.A. (2015). Equine Alphaherpesviruses, in: Robinson's Current Therapy in Equine Medicine. Elsevier Inc., pp. 158-161.

Hussey G.S., Goehring L.S., Lunn D.P., Hussey S.B., Huang T., Osterrieder N., Powell C., Hand J., Holz C. Slater J. (2013). Experimental infection with equine herpesvirus type 1 (EHV-1) induces chorioretinal lesions. Vet Res. 44:1-15.

Kydd J.H., Townsend H.G.G. Hannant D. (2006). The equine immune response to equine herpesvirus-1: The virus and its vaccines. Vet Immunol Immunopathol. 111:15-30.

Lunn D.P., Horohov D.W., Osterrieder K. Pusterla N. (2009). EHV-1 Consensus Statement. J Vet Int Med. 23:450-461.

Patel J.R. Haldens, J. (2005). Equine herpes 
viruses $1(\mathrm{EHV}-1)$ and $4(\mathrm{EHV}-4)-$ Epidemiology, Disease and Immunoprophylaxis: A brief review. Vet $J$ 170:14-23.

Pustrela N. and Hussey GS. (2014). Equine herpes virus 1 myeloencephalopathy. Vet Clin North Am Equine Pract 30:489-506.

Quinlivan M., Dempsey E., Ryan F., Arkins S. Cullinane A. (2005) Realtime reverse transcription PCR for detection and quantitative analysis of equine influenza virus. J Clin Microbiol. 43:5055-7.

Reed S.M., Toribio R.E. (2004). Equine Herpes Virus 1 and 4. Vet Clin North Am Equine Prac 20:631-642.

Slater, J. (2014). Equine Herpesviruses, in: Equine Infectious Diseases. Elsevier Inc., p. 151-168.e8.

Smith K.C. Borchers K. (2001) A study of the pathogenesis of equid herpesvirus-1 (EHV-1) abortion by DNA in-situ hybridization. J Comp Pathol 125:304310.

Swerczek T.W., Dennis S.M. (2006). Equine Herpesvirus Infections, in: Current Therapy in Large Animal Theriogenology: Second Edition.
Elsevier Inc., pp. 176-180.

Telford E.A., Watson M.S., Perry J., Cullinan A.A. Davison A.J. (1998). The DNA sequence of equine herpesvirus 4. J Gen Virol 79:1197-1203.

van Mannen C. (2002) Equine Herpes virus 1 and 4 infections: an update. Vet Quarterly. 24:57-78.

Vandekerckhove A.P., Glorieux S., Gryspeerdt A.C., Steukers L., Van Doorsselaere J., Osterrieder N., Van de Walle G.R. Nauwynck H.J. (2011). Equine alphaherpesviruses (EHV-1 and EHV-4) differ in their efficiency to infect mononuclear cells during early steps of infection in nasal mucosal explants. Vet Microbiol. 152:21-28.

Wilsterman S., Soboll-Hussey G., Lunn D.P., Ashton L.V., Callan, R.J., Hussey S.B., Rao S., Goehring, L.S., (2011). Equine herpesvirus-1 infected peripheral blood mononuclear cell subpopulations during viremia. Vet Microbiol. 149:40-47.

Wood J., Smith K.C., Daly J.M. Newton J.R. (2007). Viral infections of the equine respiratory tract, Equine Respiratory Medicine and Surgery. Elsevier Ltd.

\section{How to cite this article:}

Jignesh Vala, Manish Patel, Saurabh Parmar, Suresh Mavadiya and Sudhir Mehta. 2020. Equine Herpes Virus 1 and 4 Infections in equines: A Review. Int.J.Curr.Microbiol.App.Sci. 9(07): 100-105. doi: https://doi.org/10.20546/ijcmas.2020.907.011 